\title{
Unsupervised method of word sense disambiguation for real time associated word identification in human-robot interaction
}

\author{
Sukjae Choi \\ Humanitas BigData Research Center, \\ Kyung Hee University, \\ 26 Kyunghee-daero, Dongdaemun-gu, \\ Seoul, 130-701, Korea \\ Fax: +8229610515 \\ Email: sjchoi@khu.ac.kr

\section{Ohbyung Kwon*} \\ School of Management, \\ Kyung Hee University, \\ 26 Kyunghee-daero, Dongdaemun-gu, \\ Seoul, 130-701, Korea \\ Fax: +82 29610515 \\ Email: obkwon@khu.ac.kr \\ ${ }^{*}$ Corresponding author
}

\begin{abstract}
This paper presents a system architecture and algorithm for the disambiguation problem in human-robot interaction. Currently, when we have a communication with robot, there are ambiguity problems which lead to a misunderstanding. Conventional methods only identify ambiguity in limited ways and in few contexts due to the cost of doing so. The proposed method using real Hangul input object (RHINO) cloud identifies ambiguous words, phrases and sentences in many contexts and suggests appropriate alternatives. And by calculating the frequency of an ambiguous word, an associated word and the theme we can obtain the associated strength. The theme which has the biggest strength is the meaning of the ambiguous word. This process reflects the fluctuation of associated words' social cultures because it searches words in real time.
\end{abstract}

Keywords: human-robot interaction; WSD; word sense disambiguation; social robot; disambiguation problems; solver base; sentiWordNet; RHINO cloud.

Reference to this paper should be made as follows: Choi, S. and Kwon, O. (2016) 'Unsupervised method of word sense disambiguation for real time associated word identification in human-robot interaction', Int. J. Advanced Media and Communication, Vol. 6, No. 1, pp.20-38.

Biographical notes: Sukjae Choi is a Research Professor at Humanitas BigData Research Center, Kyung Hee University, Korea. He received his BA, MA and PhD at Korea University, 1999, 2001, 2008, respectively. He worked at Language Technologies Institute (LTI) at Carnegie Mellon University as a Visiting Researcher for English-Korean Machine Translation project. $\mathrm{He}$ also worked at Language Engineering Institute at Yanbian University of 
Science and Engineering for Korean-Chinese Machine Translation system. He taught Korean language and grammar at Sungshin Women's University as an Assistant Professor. His current includes NLP, big data analytics and information retrieval.

Ohbyung Kwon is presently a Full Professor at School of Management, Kyung Hee University, Korea, where he initially joined in 2004. In 2002, he worked Institute of Software Research International (ISRI) at Carnegie Mellon University to perform a project on context-aware computing, web service and semantic web. He received his BA at Seoul National University and $\mathrm{PhD}$ at KAIST in 1988 and 1995, respectively. He was also an Adjunct Professor at San Diego State University (SDSU). His current research interests include data science, context-aware services, e-commerce and DSS. He has presented various papers in leading information system journals including Journal of Management Information System, Decision Support Systems, Journal of Computer Information Systems and International Journal of Information Management.

This paper is a revised and expanded version of a paper entitled 'Method of disambiguate problem for real-time review in human-robot interactions' presented at 2015 International Conference on Platform Technology and Service, Jeju, Korea, 26-28 January, 2015.

\section{Introduction}

With knowledge and technology of natural language processing and voice recognition, human can have a conversation with robots. However, dialogue between humans and robots is still very simple and straightforward than actual human-to-human conversations, mainly due to robots' lack of information and inability to interpret that information contextually. Robots can understand simple questions with 'yes or no' answers, and can convey clear and simple messages; however, they are unable to detect exact meanings from more complicated phrases or make subtle distinctions of meaning.

Even for people, language analysis can be very difficult because meanings are often ambiguous and multiple. Unless the context of the conversation is understood, interpretation will not be exact. This difficulty with language interpretation, which has been called word sense disambiguation (WSD), is a long-studied problem in the natural language processing community. It is important especially in research on information extraction and text mining (Ginter et al., 2004). Consider, for example, the word 'apple'. Because this word could refer to the name of a company or a fruit, it is not easy to discern its exact meaning when it appears in a sentence for an untrained entity such as a robot.

Imperfect or erroneous interpretation of conversation results from failure to detect ambiguity and vagueness. Ambiguity happens when an expression can be interpreted in two or more different ways, while vagueness occurs when the exact meaning of an expression cannot be determined. Ambiguity and vagueness are often used to make communication more economical. A speaker who assumes that the listener already understands the context of the conversation may not provide complete information, but may instead simplify his or her message. For robots, who lack the ability to cope with ambiguity and vagueness, conversations based on this assumption are too difficult. The result is an unnatural response from the robot to the human speaker. 
Everyday conversation is a non-structured type of language that includes certain words or surface forms. Disambiguation involves selection of one of these surface forms from among all potential candidates. A typical example can be found in research on uniform resource identifiers (Mendes et al., 2011) using the latent Dirichlet allocation method to detect ambiguity (Blei et al., 2003; Kim et al., 2014). However, previous research focused on nouns; thus, ambiguity of larger units of meaning such as phrases and sentences remain a problem. Very often, human conversation about such diverse and complex topics as education, tours, orders or healthcare includes large amounts of ambiguous language. Robots lack mechanisms to cope with this, and therefore they cannot easily analyse speakers' emotions and intention. As a result, the quality of service provided by robots may be reduced.

In this paper, ambiguous expressions and detection of precise meanings by service robots are considered in many contexts. An experiment is conducted in which a social robot is given the opportunity to interpret ambiguous expressions, and its performance is evaluated.

\section{Word sense disambiguation}

A word sense disambiguation (WSD) problem happens when a word or set of words has multiple potential meanings. To solve WSD problems, various potential solutions have been suggested; these are classified into supervised and unsupervised algorithms. Supervised algorithms require readymade information sets constructed mainly through manual effort. Wilks (1972) used a semantic hierarchy including 80 semantic features such as HUMAN, MACHINE and MOVE. For subjects that can be interpreted in two or more different ways, each possible interpretation has its own semantic feature. By checking the verb, the appropriate semantic feature can be chosen. But choosing enough semantic features and building a semantic hierarchy is not easy, as evidenced when this method was applied within a small English-French machine translation system (Wilks, 1973).

Small $(1979,1980)$ asserted that words contain all the information that needs to be interpreted in a sentence. According to him, not even grammar is needed, because words reflect the organisation of human knowledge in the form of language. However, in order to be true, every word in a given system (what Small called the 'word expert') must be considered very carefully because all words should contain all the information it relates with. While it is true those words are the most important and prominent element in language, if there are a least amount of grammar the whole system will be more concise and portable.

Hirst (1987) implemented a multi-level system which combines grammar, the lexicon, semantics, a parser and knowledge representation modules. In contrast to Small's word-focused, one-module system, Hirst distributed the disambiguation process to multiple modules. As a result, interpretation of words was completed gradually, like Polaroid photos; therefore, he called them 'Polaroid words'. Unfortunately, including too many levels for one purpose makes the system too complex, and the need for hand-selected information makes completion uncertain.

McRoy (1992) and Stevenson and Wilks (1999) also created a system which combines several knowledge bases of words. McRoy's system includes a corpus, a concept hierarchy and collocation sets. The difference between the system and that of 
Hirst (1987) is in the scoring method. Every knowledge base is assigned a score from -10 to +10 . However, this program has problems of inaccuracy. Stevenson and Wilks (1999) integrated different sources of knowledge successfully. They reported a disambiguation rate of 90-94\%, indicating considerable success.

Yarowsky (1994) used a decision list consisting of words chosen according to the frequency with which they appear with a particular meaning. For ambiguous words, the system checks the decision list of leading and trailing words and decides the most frequent sense of these words. There are two problems with this method. First, frequency information is not available for some words; in such cases, this method produces no results. Second, building a decision list is very difficult. For databases including a vast number of words, to find and record their frequency according to sense cannot be completed easily.

While the algorithms described above all require some manual effort, unsupervised algorithms support autodisambiguation. Lesk (1986) suggested one such autodisambiguating algorithm. Generally, words do not stand alone. Instead, multiple companion words go together in a sentence because they share some common properties. Lesk (1986) found these common properties in dictionary definitions. Examining various definitions of words, he assumed that the appropriate definition would feature the most common words among others in the same sentence. Using this method, he reported an encouraging 70\% success rate. However, Lesk's algorithm was not always successful. A sentence like 'The astronomer married the star' will not be properly interpreted using Lesk's algorithm. Related with this, Waltz and Pollack (1985) proposed case frames, semantic priming and syntactic preferences as important elements in determining meaning. McCarthy et al. (2004) used a thesaurus to find a distributional similarity score between the words.

Brown et al. (1991) used a bilingual corpus. If a word has several meanings, when it is translated into another language, the translated words may also differ in meaning. They found cues in the first and second words to the left and right of ambiguous words in his examination of translated words in a target language corpus. This research was used to improve the machine translation system.

In other research, two new constraints were suggested. Gale et al. (1992) argued that words that have one sense in one discourse may have another meaning in another discourse. In an experiment with well-written discourse, $98 \%$ of words were found to have the same sense in one discourse. Furthermore, Yarowsky (1993) also argued that words may have one sense in one collocation. In several experiments, it was found that words do not yield different senses in the same collocation. Then, Yarowsky (1995) suggested an unsupervised learning algorithm with these two constraints. From the dictionary definitions of various words, disambiguation properties were determined in observing one sense per discourse and one sense per collocation. Yarowsky (1995) reported a success rate of $90-96 \%$ in terms of disambiguation, depending on the definition of the word.

After 2000, graph-based methods are well known in this area. Graph-based method uses node for a concept and edges for relations between concepts (Mihalcea, 2005; Navigli and Lapata, 2010). These methods take into account all combined senses and have a strong point on analysing the interrelations of them.

Graph-based method is currently still developing. Wessam et al. (2013) used medical thesaurus as a knowledge base and it took advantage of expertise. Eneko et al. (2014) presented the model which has random walks over relations in WordNet, therefore it uses 
full resource of WordNet's knowledge. And by changing language specific WordNet it can be easily ported to other languages.

Recently, there is an effort also linking WSD's result to entity linking as in Andrea et al. (2014) and Yongbo et al. (2014). After all, WSD's research will greatly contribute to AI area with other NLP areas successful outcome. To increase the quality of human-robot interaction, the robot should have the ability to determine which of various senses of a word or a set of words are invoked in a given context. The ambiguity of natural language can be classified as lexical, structural, scope related or pragmatic. Among these, lexical, structural and scope-related ambiguity result from language expressions, and hence are categorised as internal language ambiguity. In many cases, what causes ambiguity in a sentence can be easily identified.

\subsection{Lexical ambiguity}

Lexical ambiguity occurs when one word has several meanings. If we do not consider the context at all, we cannot decide the word's meaning. Here are some examples with possible interpretations in parentheses.

- He gave me a hand (applause/help).

- She has a big mouth (wide mouth/is talkative).

\subsection{Structural ambiguity}

Structural ambiguity occurs when the sentence structure has several interpretations. We can classify this type of ambiguity further as modifier ambiguity and object ambiguity. Examples and possible interpretations are as follows.

I want cold coffee and tea.

- cold coffee and general tea

- cold coffee and cold tea

I came here to see my sister with my wife.

- I and my wife came here to see my sister.

- I want to see my sister and my wife, who is with my sister.

\subsection{Scope ambiguity}

If a sentence includes a quantifier or a negative, scope-related ambiguity may be present. Some examples and possible interpretations follow.

All students in this class must have a laptop.

- All students must have a laptop (as most people have them these days).

- We need at least one laptop for all students. 
I said I did not want that kind of car in this weather.

- I said when the weather was like this, I did not want that kind of car.

- I said I did not want to drive that kind of car, especially in this weather.

\subsection{Pragmatic ambiguity}

Pragmatic ambiguity is hard to identify. It mainly results from the background of the conversation rather than from a specific word or expression. Thus, this is called external language ambiguity. In many cases, information as to time, location and schedule can aid in interpretation. Some examples are below.

A: Can you come here now?

B: I have a class until 3 p.m.

- I just informed you about my schedule.

- You know what time it is. So you understand I cannot go.

\section{Method}

The proposed system architecture is a new approach for solving the problems inherent in human-robot interaction. This method enables the detection of ambiguous words and phrases in real time. If a human client mentions his or her perceptions (e.g., about the usefulness of an object), feelings (e.g., intimate, close, familiar, etc.) or intentions (e.g., satisfaction, adoption) to the service robot, then the robot recognises the human speech and stores it as unstructured sentences. Then the robot's engine conducts a series of activities in order to respond to the client: stemming, identifying ambiguity, sentiment analysis and dialogue generation. During the sequence, the robot accesses a cloud system, real Hangul input object (RHINO) cloud, to aid in linguistic operations. RHINO cloud is able to identify appropriate meanings by searching for sentences containing ambiguous words using Google in real time. The results of these searches reflect the social and cultural influences surrounding words in an unsupervised way, updating this information automatically without human intervention. The whole process is shown in Figure 1.

Figure 1 System architecture (see online version for colours)

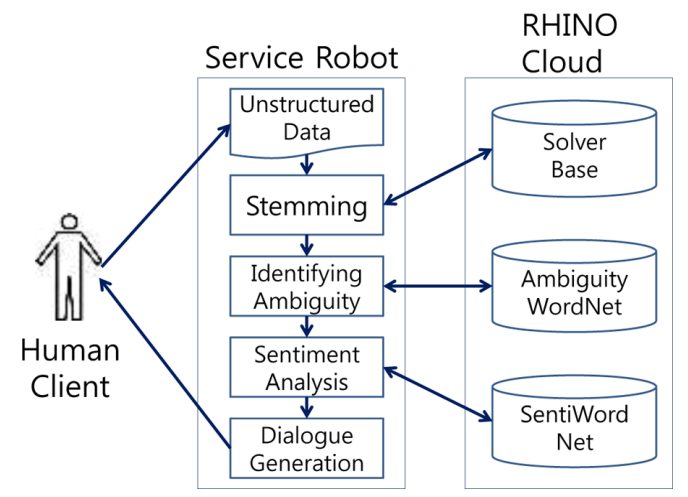




\subsection{Solver base}

Any morphology, which includes a stem and ending, is regarded as an object in the system. An object as a solver in the solver base contains a method with a name identical to the object's name. For example, an object 'Eun' has the following method: The Korean suffix 'Eun', which is recorded in the Korean alphabet. This method allows us to cope with exceptional situations and retain structural safety. The system engine for analysing phrases in unstructured data improves accuracy.

In the solver base, the morphological analyser RHINO plays a major role. It contains a dynamic dictionary to identify morphology according to the context in which it is used. RHINO runs with 270,000 stems and 85,000 ending expressions.

\subsection{WordNet}

To support the identification of ambiguity in a sentence, WordNet is established remotely and then accessed by a service entity (e.g., a social robot or an agent) after authorisation. To utilise WordNet, the system must first choose the ambiguous words and save them in a list. Table 1 shows some examples of ambiguous words.

The crawler then collects example sentences containing ambiguous words from online documents. After analysing words morphologically, the system saves common cooccurring words. These words may have strong connections with the ambiguous words. Example sentences also can be found in the user interface.

Table 1 Ambiguous words

\begin{tabular}{lll}
\hline Word & Meaning 1 & Meaning 2 \\
\hline Bear & Animal & Endure \\
Bow & Weapon & Bend \\
Bill & Account & A law bill \\
Change & Alteration & Money \\
Class & Stratum & Lesson \\
Paper & News & Grammatical entity \\
Degree & Course & Temperature \\
Even & Flat & Reference to numbers \\
Express & Fast & Show \\
Good & Related to goodness & Product \\
Mind & Heart & Avoid \\
Yield & Produce & Give way \\
\hline
\end{tabular}

\subsection{SentiWordNet}

SentiWordNet provides lists of emotion words. Each emotion word is given a positive or negative score. Shaver et al. (1987) classified 135 selected English emotion words and classified them into six categories. Kim (2004) did the same with 494 selected Korean emotion words. By storing these words, the system can show the speaker's current emotional state and the degree to which he or she is feeling this emotion. 


\subsection{Removing stop words}

For the purposes of analysis, sentences must be simplified. Because papers, prepositions, simple function words (e.g., is, are, do and should), conjunctions and punctuation marks do not provide important information, they are removed from the text. This process is known as 'preprocessing'. Using the example of the sentence containing the ambiguous word 'apple', the results may be as shown in Table 2.

Table 2 Search results for ambiguous words

[1] Apple designs the Mac, along with OS and iWork

[2] Change the date range and chart type and compare Apple Inc. against other companies

[3] AAPL news, historical stock charts, analyst ratings, financials, and today's Apple Inc. stock price

[4] Check out the latest Apple news on CNET, featuring developments on the iPhone, iPad, Macbooks, OS X and ...

[5] Apple introduced its second-generation iPad Air on October 16, with pre-orders beginning on 17 October

[6] Apple scanned for music purchased from rival services such as Amazon and forced users to delete all music ...

[7] The apple tree is a deciduous tree in the rose family best known for its sweet, pomaceous fruit, the apple

[8] I am going to tell you how to grow apple trees from seed

[9] Recent research has shown that intake of apples in their whole food form can significantly lower many of ...

[10] Apples are often eaten raw, but can also be found in many prepared foods (especially desserts) and drinks

[11] A tasty combination of crunchy cauliflower, sweet apple, and creamy, salty blue

Pre-processing of the results in Table 2 is recorded in Table 3.

Table 3 Preprocessing of search results

[1] Apple designs Mac OS iWork

[2] Change date range chart type compare Apple Inc. companies

[3] AAPL news historical stock charts analyst ratings financials today Apple Inc. stock price

[4] Check latest Apple news CNET featuring developments iPhone iPad Macbooks OS X much more

[5] Apple introduced second-generation iPad Air October pre-orders October

[6] Apple accused lawyers court case deleting songs rival services some iPods past decade

[7] Apple scanned music purchased rival services Amazon forced users delete music iPods claimed

[8] Apple tree Malus domestica deciduous tree rose family best sweet pomaceous fruit apple

[9] Tell how grow apple trees seed

[10] Recent research shown intake apples whole food significantly lower many blood fats

[11] Apples eaten raw many prepared foods especially desserts drinks 


\subsection{Building the associated words set}

To identify the meanings of ambiguous words, the system must find associated words. Associated words are identified by morphological analysis of the sentences containing ambiguous words. If some words frequently cooccur with the ambiguous words, they can be classified as associated words. The associated words set is then constructed according to the following procedure.

- collect online documents containing the theme of ambiguous words

- $\quad$ analyse the documents and determine the frequency of each word

- $\quad$ obtain the exposure ratio of each word for the theme document $\left(r_{j}^{T}, j=1, \ldots, N\right)$

- collect online documents containing the general theme

- $\quad$ analyse the documents and determine the frequency of each word

- obtain the exposure ratio of each word for the general theme document $\left(r_{j}^{G}, j=1, \ldots, N\right)$.

- $\quad$ obtain the relative ratio of three of $\operatorname{six}\left(r_{j}^{T} / r_{j}^{G}\right)$

- $\quad$ select words which have $r_{j}^{T}$ (greater than the threshold value $\theta_{1}$ ) or $r_{j}^{T} / r_{j}^{G}$ (greater than the threshold value $\left.\theta_{2}\right)$ with the associated words set $\Xi\left(\Xi=\left\{\xi_{1}, \ldots \xi_{M}, M \leq N\right\}\right)$.

\subsection{Choosing the appropriate themes}

When the associated words set is constructed, the most appropriate themes for each ambiguous word must be chosen. To identify the correct meaning, the system should know that the ambiguous word $(W)$ accompanies certain associated words $(A)$ based on a given theme $(T)$. The very strong semantic joint position will reveal which one is appropriate, and the chosen theme will be directly connected to the meaning of the ambiguous word.

To calculate the equation, certain variables must first be defined (see Table 4).

Table 4 Variables used for calculating appropriate meanings

\begin{tabular}{ll}
\hline Notation & Meaning \\
\hline$f_{w}$ & Search results for an ambiguous word (W) (e.g., 'apple') \\
$f_{T}$ & Search results for the theme (T) of the ambiguous word (e.g., company, fruit) \\
$f_{A}$ & $\begin{array}{l}\text { Search results for words associated (A) with the ambiguous word } \\
\text { (e.g., Mac, tree) }\end{array}$ \\
$f_{W}^{*} T$ & Search results for the ambiguous word and its theme (e.g., apple, fruit) \\
$f_{W}^{*} T^{*} A$ & $\begin{array}{l}\text { Search results for the ambiguous word, its associated words, and its theme } \\
\text { (e.g., apple, Mac, company) }\end{array}$ \\
\hline
\end{tabular}

Then the system calculates the strength of the association for every theme. To decide the most appropriate theme from the various candidate themes, the system must find the joint position of the ambiguous word, an associated word, and the theme. In Figure 2, position 
' $A$ ' is connected with three components: an ambiguous word, an associated word and the theme. The larger the area ' $A$ ', the closer the relation. Evidently, the connected theme $(T)$ is the one we are looking for.

Figure 2 The relationships among the ambiguous word, associated word, and theme (see online version for colours)

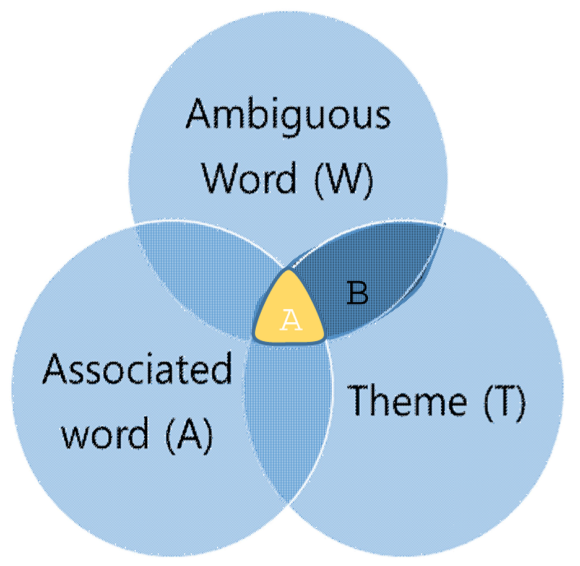

The equation associated with Figure 2 is defined as follows. We can say that $f_{W^{*} A^{*} T}$ as an associated ratio for a theme of an ambiguous word and an associated word.

$$
f_{W * A * T} / f_{W * T}=\sum_{i=1}^{M} f_{W * \xi_{i} * T} / f_{W * T} .
$$

For a final decision on the meaning of the ambiguous word, the system calculates the $f_{W^{*} A^{*} T}$ for each candidate theme of the ambiguous word and selects the theme with the highest frequency, as below.

$$
\operatorname{Pr}(t \mid W, A)=f_{W * t * A} / \sum_{\forall t \in T} f_{W * t * A} .
$$

For example, if there is an input sentence, 'Apple designs Mac OS X iLife iWork', and we want to know the meaning of the word 'apple', the system finds the words associated with the word 'apple' in this sentence. In this sentence, 'Mac' is associated with the word 'apple', so the system searches for the theme by identifying sentences that include this ambiguous word, the ambiguous word 'Mac' and the two themes 'electronic' and 'fruit'. Below are the two words groups which result.

1 Apple Mac company

2 Apple Mac fruit.

The search results include 95 and 13 million entries for the company and the fruit, respectively. Because the results for (1) are greater than those for (2), we can decide that the meaning of 'apple' would be 'company'. This decision reflects the fluctuating nature of social culture because the system searched online in real time.

If the resulting number of entries is too low for comparison, searches can be done with more associated words. In the sentence above, the words 'designs', 'OS' and 
'iWork' may also yield some results. By searching for associated words, we can be very sure that the right theme has been identified. This process is described in Table 5.

At the end of the analysis, we can say that the word 'apple' in the example sentence means 'company' with a probability of $61.3 \%$.

Table 5 Associated words search results

\begin{tabular}{lcc}
\hline Words & Company $(A=1)$ & Fruit $(A=2)$ \\
\hline Apple designs & 174 & 48.8 \\
Apple Mac & 66 & 15.4 \\
Apple OS & 49.1 & 5.12 \\
Apple iWork & 8.65 & 3.62 \\
Total & 297.75 & 72.94 \\
Apple & 418 & 162 \\
Adjusted rate & 0.27 & 0.72 \\
$R=$ Total $\times$ Adjusted rate & 83.16 & 52.56 \\
$\operatorname{Pr}(A)=R(A=i) /(R(A=i)+R(A=j)$ & 0.613 & 0.387 \\
Decision & Apple is a company (Correct)
\end{tabular}

\section{Experiment}

\subsection{Sampling and procedure}

For the evaluation, we used the test set (see Table 6) provided by Yarowsky (1995), which has been utilised in many similar studies. The senses were selected from WordNet. Rather than using available sense-tagged corpora such as http:/www.itri.brighton.ac.uk/ events/senseval, we used our prototype system and Google search API: an open big data and open source.

Because there are very few studies in which the correct meaning is automatically identified, it is difficult to perform a comparative study. For example, Yarowsky (2000), in one representative study, reported an accuracy rate of $95 \%$ for the words 'plant', 'tank', 'poach' and 'palm'. However, because this research was built on the premise of a reserve decision list, a simple direct comparison is difficult to make. Therefore, we compared the accuracy between the following methods: maximum likelihood, Senseval, and our proposed method. We adopted accuracy as a metric, because the accuracy depends on how difficult the disambiguation task is according to the number of senses and sense proximity.

Table 6 Yarowsky (1995) test set

\begin{tabular}{llll}
\hline Word & Theme & Word & Theme \\
\hline Plant & Living/factory & Bass & Fish/music \\
Tank & Vehicle/container & Motion & Legal/physical \\
Poach & Steal/boil & Crane & Bird/machine \\
Palm & Tree/hand & & \\
\hline
\end{tabular}




\subsection{Results}

Appendix A and Table 7 shows the results of the analysis comparing the three methods. The results reveal that the proposed method obviously outperforms the maximum likelihood and Senseval methods for all test words except 'palm'. In many cases, the proposed method shows a high accuracy up to 0.93 and the average accuracy is 0.74 . Compared to that, the other two methodologies have lower accuracy mostly around 0.50 , and the average is 0.48 and 0.59 , respectively. Just in case of 'palm' the proposed method does not outperform, but in that case also it has equal accuracy with Senseval.

In addition, we performed a $t$-test to determine the statistical significance of the results presented in Table 7 . As a result, the proposed method shows the statistical superiority of our method compared to other methodologies: maximum likelihood $(p<0.01)$ and Senseval $(p<0.05)$, as shown in Table 8 . The result suggests that the proposed method is usable enough to be adopted by legacy text mining tools.

Table 7 Comparison of results obtained using the three methods

\begin{tabular}{lccccc}
\hline & \multicolumn{3}{c}{ Proposed method } & Maximum likelihood & $\begin{array}{c}\text { Senseval } \\
\text { Word }\end{array}$ \\
\cline { 2 - 5 } Apcuracy & Correct & Incorrect & Accuracy & 0.45 & 0.54 \\
Plant & 9 & 2 & 0.82 & 0.50 & 0.50 \\
Tank & 13 & 1 & 0.93 & 0.50 & 0.57 \\
Poach & 9 & 5 & 0.64 & 0.50 & 0.57 \\
Palm & 9 & 5 & 0.64 & 0.50 & 0.86 \\
Bass & 12 & 2 & 0.86 & 0.50 & 0.71 \\
Motion & 12 & 2 & 0.86 & 0.50 & 0.50 \\
Crane & 13 & 6 & 0.57 & 0.50 & 0.64 \\
Spring & 11 & 1 & 0.93 & 0.33 & 0.43 \\
Total & 96 & 9 & 0.55 & 0.48 & 0.59 \\
\hline
\end{tabular}

Table $8 \quad T$-test results

\begin{tabular}{lcc}
\hline & $\begin{array}{c}\text { The average difference } \\
\text { in accuracy }(\%)\end{array}$ & $t$-value \\
\hline Proposed vs. maximum likelihood & 26.8 & $2.306^{* *}$ \\
Proposed vs. Senseval & 15.2 & $2.119^{*}$ \\
\hline
\end{tabular}

$* p<0.05, * * p<0.01$.

\section{Conclusion}

\subsection{Contributions}

This study is the first to consider phrases and context in coping with disambiguation problems. Including these makes the analysis more efficient for the following two reasons. First, disambiguation is successful even with very little prerequisite data using our suggested algorithm. Previous methods required many kinds of semantic tags, rules, 
multilevel processes or decision trees. These make the system very complicated and the job very difficult. Comparatively speaking, the suggested algorithm, which requires only associated words that are easily and automatically acquired, provides better results.

Second, by searching web documents in real time, the system provides a continuous reflection of current social culture. The words used in sentences on a specific theme vary considerably according to the times and rapidly changing social culture. When sentences have been analysed only once and the resulting information used only in a static way, the results will be inadequate because key associated words will be lacking. Using the suggested algorithm allows the system to include currently used expressions, with the result of more successful outcomes.

Furthermore, based on the results from our ambiguity identification and sentiment analyses, two types of response, sympathy and confirmation, can be generated for longer conversations, which is often necessary in the context of social service robots. If the robot detects an ambiguity in the conversation and can identify the exact meaning, it may be able to generate dialogue indicating sympathy (e.g., "Yes, you are really impressed by the movie"). If the robot detects ambiguity in the conversation only, then it may generate a sentence like, "You seem to be impressed by the movie, don't you?" If 'apple' refers to a company name, then the robot may react by saying, "It is a nice company, isn't it?", while if it means 'fruit', it may react by saying, "It's delicious, isn't it?".

\subsection{Future research}

In future research, we will examine more ambiguous words and apply our proposed disambiguation method. We also intend to extend the suggested algorithm to other languages. Although we utilised test words from the literature in this study, the proposed method should be utilised with other words to ensure its generalisability.

\section{Acknowledgement}

This work was supported by the National Research Foundation of Korea Grant funded by the Korean Government (NRF-2014S1A5B8060940).

\section{References}

Andrea, M., Alessandro, R. and Roberto, N. (2014) 'Entity linking meets word sense disambiguation: a unified approach', Transactions of the Association for Computational Linguistics, Vol. 2, pp.231-244.

Blei, D., Ng, A. and Jordan, M. (2003) 'Latent Dirichlet allocation', Journal of Machine Learning Research, Vol. 3, pp.993-1022.

Brown, P., Della Pietra, S., Della Pietra, V. and Mercer, R. (1991) 'Word-sense disambiguation using statistical methods', ACL-91: Proceedings of the 29th Meeting of the Association for Computational Linguistics, Berkeley, USA, pp.264-270.

Eneko, A., Oier, L. and Aitor, S. (2014) 'Random walks for knowledge-based word sense disambiguation', Computational Linguistics, Vol. 40, No. 1, pp.57-84.

Gale, W., Church, K. and Yarowsky, D. (1992) 'One sense per discourse', HLT-91: Proceedings of the Workshop on Speech and Natural Language Workshop, NY, USA, pp.233-237. 
Ginter, F., Boberg, J., Jarvinen, J. and Salakoski, T. (2004) 'New techniques for disambiguation in natural language and their application to biological text', Journal of Machine Learning Research, Vol.5, pp.605-621.

Hirst, G. (1987) Semantic Interpretation and the Resolution of Ambiguity, Cambridge University Press, Cambridge.

Kim, E.Y. (2004) The Study of Korean Emotional Words, PhD Thesis, Chonnam National University, Korea.

Kim, J., Kim, Y., Hahm, Y., Hwang, D. and Choi, K. (2014) 'LDA-based approach for URI disambiguation and error reduction', Proceedings of the 26th Conference on Hangul and Korean Language Information Processing, Chuncheon, Korea, pp.107-111.

Lesk, M. (1986) 'Automatic sense disambiguation using machine readable dictionaries: how to tell a pine cone from an ice cream cone', Proceedings of ACM SIGDOC Conference, Toronto, Canada, pp.24-26.

McCarthy, D., Koeling, R., Weeds, J. and Carroll, J. (2004) 'Finding predominant word senses in untagged text', ACL-04: Proceedings of the 42nd Meeting of the Association for Computational Linguistics, Barcelona, Spain, pp.279-286.

McRoy, S. (1992) 'Using multiple knowledge sources for word sense disambiguation', Computational Linguistics, Vol. 18, No. 1, pp.1-30.

Mendes, P.N., Jakob, M., García-Silva, A. and Bizer, C. (2011) 'DBpedia spotlight: shedding light on the web of documents', ACM 2011: Proceedings of the 7th International Conference on Semantic Systems, pp.1-8.

Mihalcea, R. (2005) 'Unsupervised Large-vocabulary word sense disambiguation with graph-based algorithms for sequence data labeling', Proceedings of the Conference on Human Language Technology and Empirical Methods in Natural Language Processing, (HLT'05), Morristown, NJ, pp.411-418.

Navigli, R. and Lapata, M. (2010) 'An experimental study of graph connectivity for unsupervised word sense disambiguation', IEEE Transactions on Pattern Analysis and Machine Intelligence, Vol. 32, No. 4, pp.678-692.

Shaver, P., Schwarth, J., Kirson, D. and O'Connor, C. (1987) 'Emotion knowledge: further exploration of a prototype approach', Journal of Personality and Social Psychology, Vol. 52, No. 6, pp.1061-1086.

Small, S. (1979) 'Word expert parsing', ACL-79: Proceedings of the 17th Annual Meeting on Association for Computational Linguistics, Stroudsburg, PA, USA, pp.9-13.

Small, S. (1980) Word Expert Parsing: A Theory of Distributed Word-Based Natural Language Understanding, $\mathrm{PhD}$ Thesis, University of Maryland, Maryland, USA.

Stevenson, M. and Wilks, Y. (1999) 'Combining weak knowledge sources for sense disambiguation', Proceedings of the 16th International Joint Conference on Artificial Intelligence, Stockholm, Sweden, pp.884-889.

Waltz, D. and Pollack, J. (1985) 'Massively parallel parsing: a strongly interactive model of natural language interpretation', Cognitive Science, Vol. 9, pp.51-74.

Wessam, G.E., Osmar, R.Z. and Mohammad, E.H. (2013) 'Unsupervised graph-based word sense disambiguation of biomedical documents', IEEE 15th International Conference on e-Health Networking, Applications and Services (Healthcom), pp.649-652.

Wilks, Y. (1972) Grammar, Meaning and the Machine Analysis of Language. Routledge, London.

Wilks, Y. (1973) 'The stanford MT project', in Rustin, R. (Ed.): Natural Language Processing, Algorithmics Press, New York.

Yarowsky, D. (1993) 'One sense per collocation', Proceedings of the ARPA Human Language Technology Workshop, Princeton, USA, pp.266-271.

Yarowsky, D. (1994) 'Decision lists for lexical ambiguity resolution: application to accent restoration in Spanish and French', Proceedings of the 32nd Annual Meeting of the Association for Computational Linguistics, Las Cruces, USA, pp.88-95. 
Yarowsky, D. (1995) 'Unsupervised word-sense disambiguation rivaling supervised methods', ACL-95: Proceedings of the 33rd Annual Meeting of the Association for Computational Linguistics, Cambridge, Mass., USA, pp.189-196.

Yarowsky, D. (2000) 'Hierarchical decision lists for word sense disambiguation', Computers and the Humanities, Vol. 34, Nos. 1-2, pp.179-186.

Yongbo, Y., Lizhou, Z., Kaifang, Y. and Peiquan, J. (2014) 'Named entity linking based on Wikipedia' International Journal of Database Theory and Application, Vol. 7, No. 1, pp.1-10.

\section{Appendix A. Test Examples}

*For brevity, some typical examples are reported in Tables A1-A10.

Table A1 Change date range chart type compare Apple Inc. companies

\begin{tabular}{lcc}
\hline Words & Company & Fruit \\
\hline Apple change & 186 & 67.4 \\
Apple date & 185 & 63.5 \\
Apple range & 146 & 45.8 \\
Apple chart & 47.3 & 2.26 \\
Apple type & 131 & 52.6 \\
Apple compare & 64.4 & 18.8 \\
Apple Inc. & 92.3 & 12.6 \\
Apple companies & 180 & 60.2 \\
Total & 1032 & 323.16 \\
$R=$ Total $\times$ Adjusted rate & 288.25 & 232.90 \\
$\operatorname{Pr}(A)=R(A=i) /(R(A=i)+R(A=j)$ & 0.553 & 0.447 \\
Result & Apple is company (Correct) \\
\hline
\end{tabular}

Table A2 AAPL news historical stock charts analyst ratings financials today Apple Inc. stock price

\begin{tabular}{lcc}
\hline Words & Company & Fruit \\
\hline Apple AAPL & 1.85 & 0.182 \\
Apple news & 321 & 70.4 \\
Apple historical & 35.6 & 11.4 \\
Apple stock & 153 & 25.7 \\
Apple charts & 25.8 & 6.36 \\
Apple analyst & 41.6 & 38.3 \\
Apple ratings & 51.9 & 10.6 \\
Apple financials & 2.06 & 4.55 \\
Apple today's & 61.3 & 19.2 \\
\hline
\end{tabular}


Table A2 AAPL news historical stock charts analyst ratings financials today Apple Inc. stock price (continued)

\begin{tabular}{lcc}
\hline Words & Company & Fruit \\
\hline Apple Inc. & 93.4 & 12.7 \\
Apple stock & 153 & 25.7 \\
Apple price & 198 & 69.5 \\
$R=$ Total $\times$ Adjusted rate & 307.40 & 212.11 \\
$\operatorname{Pr}(A)=R(A=i) /(R(A=i)+R(A=j)$ & 0.592 & 0.408 \\
Result & Apple is company (Correct) \\
\hline
\end{tabular}

Table A3 Check latest Apple news CNET featuring developments iPhone iPad Macbooks OS X much more

\begin{tabular}{lcc}
\hline Words & Company & Fruit \\
\hline Apple check & 163 & 67.4 \\
Apple latest & 256 & 50.2 \\
Apple news & 321 & 70.4 \\
Apple CNET & 2.15 & 417 \\
Apple featuring & 136 & 243 \\
Apple developments & 140 & 32 \\
Apple iPhone & 138 & 28.8 \\
Apple iPad & 84.8 & 17.1 \\
Apple Macbooks & 20.6 & 1.44 \\
Apple OS & 49.1 & 5.16 \\
Apple X & 285 & 115 \\
Apple OS X & 303 & 3.39 \\
Apple much & 246 & 106 \\
Apple more & 447 & 165 \\
$R=$ Total $\times$ Adjusted rate & 512.64 & 456.57 \\
$P r(A)=R(A=i) /(R(A=i)+R(A=j)$ & 0.529 & 0.471 \\
Result & Apple is company (Correct) \\
\hline
\end{tabular}

Table A4 Apple introduced second-generation iPad Air October pre-orders October

\begin{tabular}{lcc}
\hline Words & Company & Fruit \\
\hline Apple introduced & 52.6 & 162 \\
Apple second & 196 & 62.2 \\
Apple generation & 111 & 18.8 \\
Apple second-generation & 25.1 & 1.61 \\
Apple iPad & 84.8 & 17.1 \\
Apple Air & 133 & 53.6 \\
Apple October & 164 & 45.7 \\
\hline
\end{tabular}


Table A4 Apple introduced second-generation iPad Air October pre-orders October (continued)

\begin{tabular}{lcc}
\hline Words & Company & Fruit \\
\hline Apple pre & 153 & 40.8 \\
Apple orders & 189 & 64.1 \\
Apple pre-orders & 44.7 & 4.17 \\
Apple preorders & 2.8 & 0.494 \\
$R=$ Total $\times$ Adjusted rate & 299.44 & 219.25 \\
$\operatorname{Pr}(A)=R(A=i) /(R(A=i)+R(A=j)$ & 0.577 & 0.423 \\
$\operatorname{Result}$ & \multicolumn{2}{c}{ Apple is company (Correct) } \\
\hline
\end{tabular}

Table A5 Apple tree Malus domestica deciduous tree rose family best sweet pomaceous fruit apple

\begin{tabular}{lcc}
\hline & Company & Fruit \\
\hline Apple tree & 22.6 & 15.3 \\
Apple Malus & 0.44 & 0.479 \\
Apple domestica & 0.496 & 0.42 \\
Apple deciduous & 0.356 & 0.471 \\
Apple rose & 86.3 & 37.3 \\
Apple family & 62 & 35.5 \\
Apple best & 381 & 139 \\
Apple sweet & 97 & 77.3 \\
Apple pomaceous & 0.321 & 0.126 \\
Apple fruit & 53.6 & 162 \\
$R=$ Total $\times$ Adjusted rate & 175.73 & 220.40 \\
$\operatorname{Pr}(A)=R(A=i) /(R(A=i)+R(A=j)$ & 0.444 & 0.556 \\
Result & Apple is fruit (Correct) \\
\hline
\end{tabular}

Table A6 Tell how grow apple trees seed

\begin{tabular}{lcc}
\hline & Company & Fruit \\
\hline Apple tell & 123 & 38.5 \\
Apple how & 407 & 131 \\
Apple grow & 99.3 & 25 \\
Apple trees & 10.4 & 4.94 \\
Apple seed & 8.07 & 6.75 \\
$R=$ Total $\times$ Adjusted rate & 344.97 & 533.02 \\
$\operatorname{Pr}(A)=R(A=i) /(R(A=i)+R(A=j)$ & 0.393 & 0.607 \\
$\operatorname{Result}$ & Apple is fruit (Correct) \\
\hline
\end{tabular}


Table A7 Recent research shown intake apples whole food significantly lower many blood fats

\begin{tabular}{lcc}
\hline & Company & Fruit \\
\hline Apple Recent & 201 & 65 \\
Apple research & 195 & 32.3 \\
Apple shown & 68.9 & 23.2 \\
Apple intake & 190 & 1.95 \\
Apple whole & 156 & 68.1 \\
Apple food & 184 & 131 \\
Apple significantly & 34.7 & 10.3 \\
Apple lower & 123 & 32.2 \\
Apple blood & 50.9 & 29.9 \\
Apple fats & 37.1 & 44.3 \\
$R=$ Total $\times$ Adjusted rate & 333.11 & 535.47 \\
$\operatorname{Pr}(A)=R(A=i) /(R(A=i)+R(A=j)$ & 0.384 & 0.616 \\
Result & \multicolumn{2}{c}{ Apple is fruit (Correct) } \\
\hline
\end{tabular}

Table A8 Apples eaten raw many prepared foods especially desserts drinks

\begin{tabular}{lcc}
\hline & Company & Fruit \\
\hline Apple eaten & 13.9 & 12.2 \\
Apple raw & 60.1 & 31 \\
Apple many & 238 & 85.1 \\
Apple prepared & 60.9 & 37.4 \\
Apple foods & 189 & 133 \\
Apple especially & 119 & 45.5 \\
Apple desserts & 8.57 & 23.6 \\
Apple drinks & 45.8 & 73.8 \\
$R=$ Total $\times$ Adjusted rate & 198.52 & 317.95 \\
$\operatorname{Pr}(A)=R(A=i) /(R(A=i)+R(A=j)$ & 0.384 & 0.616 \\
Result & \multicolumn{2}{c}{ Apple is fruit (Correct) } \\
\hline
\end{tabular}

Table A9 Apple muffins good old fashioned apple pie library apple offers tons ways use tasty fruit

\begin{tabular}{lcc}
\hline & Company & Fruit \\
\hline Apple muffins & 1.23 & 16.8 \\
Apple good & 271 & 134 \\
Apple old & 260 & 99.5 \\
Apple fashioned & 260 & 3.62 \\
Apple pie & 8.34 & 20.1 \\
Apple library & 79.5 & 16.3. \\
Apple offers & 121 & 41.4 \\
\hline
\end{tabular}


Table A9 Apple muffins good old fashioned apple pie library apple offers tons ways use tasty fruit (continued)

\begin{tabular}{lcc}
\hline & Company & Fruit \\
\hline Apple tons & 13.7 & 6.69 \\
Apple ways & 124 & 48.7 \\
Apple tasty & 13.8 & 22.4 \\
$R=$ Total $\times$ Adjusted rate & 311.19 & 283.11 \\
$\operatorname{Pr}(A)=R(A=i) /(R(A=i)+R(A=j)$ & 0.524 & 0.476 \\
$\operatorname{Result}$ & Apple is NOT fruit (Incorrect) \\
\hline
\end{tabular}

Table A10 Tasty combination crunchy cauliflower sweet apple creamy salty blue

\begin{tabular}{lcc}
\hline & Company & Fruit \\
\hline Apple tasty & 13.8 & 22.4 \\
Apple combination & 47.1 & 32.9 \\
Apple crunchy & 0.835 & 5.19 \\
Apple cauliflower & 0.822 & 5.19 \\
Apple sweet & 94.1 & 77.1 \\
Apple creamy & 2.1 & 20.8 \\
Apple salty & 1.06 & 1.3 \\
Apple blue & 1440 & 110 \\
$R=$ Total $\times$ Adjusted rate & 54.52 & 150.75 \\
$\operatorname{Pr}(A)=R(A=i) /(R(A=i)+R(A=j)$ & 0.266 & 0.734 \\
Result & \multicolumn{2}{c}{ Apple is fruit (Correct) } \\
\hline
\end{tabular}

